\title{
X-ray observations of the colliding wind binary WR 25
}

\author{
Bharti Arora*, Jeewan Chandra Pandey
}

Aryabhatta Research Institute of Observational Sciences (ARIES), Nainital 263002, India

\begin{abstract}
Using the archival data obtained from Chandra and Suzaku spanning over $\sim 8$ years, we present an analysis of a WN6h+O4f Wolf-Rayet binary, WR 25. The X-ray light curves folded over a period of $\sim 208$ $\mathrm{d}$ in the $0.3-10.0 \mathrm{keV}$ energy band showed phase-locked variability where the count rates were found to be maximum near the periastron passage. The X-ray spectra of WR 25 were well explained by a two-temperature plasma model with temperatures of $0.64 \pm 0.01$ and $2.96 \pm 0.05 \mathrm{keV}$ and are consistent with previous results. The orbital phase dependent local hydrogen column density was found to be maximum just after the periastron passage, when the WN type star is in front of the O star. The hard (2.0-10.0 keV) X-ray luminosity was linearly dependent on the inverse of binary separation which confirms that WR 25 is a colliding wind binary.
\end{abstract}

\section{Introduction}

Wolf-Rayet (WR) stars are evolved hot massive O-type stars, close to the end of their nuclear burning phase. It is their hot stellar winds, with velocities in the range 1000-3000 $\mathrm{kms}^{-1}$ and mass loss rates of $10^{-6}-10^{-4} M_{\odot}$ per year (Hamann et al. 2006), that characterizes the Population I WR stars. Spectroscopically, WR stars are spectacular in appearance with strong and broad emission lines $\left(\sim 10^{3}\right.$ $\mathrm{kms}^{-1}$ ). X-rays from massive stars are thought to be produced in two ways: via embedded shocks in the radiatively driven winds close to the stars and in massive binaries via shocks in the wind collision zone between two stars (Stevens et al. 1992). Therefore, in a binary system, X-ray emission might display phase-locked variability, either as a consequence of the changing wind opacity along the line of sight or as a result of variable orbital separation in the case of an eccentric binary system.

WR 25 (HD 93162) is a bright ( $\mathrm{V}=8.1)$ WR star located in the Carina Nebula region and is classified as WN6h+O4f (van der Hucht 2001). Gamen et al. (2006) studied the radial velocity profile of WR 25 and suggested that it has an eccentric binary orbit $(\mathrm{e}=0.5)$ with an orbital period of about 208 days. Also, the ratio of X-ray to bolometric luminosity of $\sim 10^{-6}$ (Seward \& Chlebowski 1982) for WR 25 is an order of magnitude higher than observed for single WR stars, suggesting it to be a very likely candidate of colliding wind binary systems. Previous X-ray studies of WR 25 were done by Raassen et al. (2003), Pollock et al. (2006), and Pandey et al. (2014). These studies are based on limited X-ray observations. Therefore, in order to investigate this system deeply, we have carried out an X-ray study using the observations made by Chandra (Weisskopf et al. 2000) and Suzaku (Mitsuda et al. 2007) of WR 25.

This paper is organised as follows. Section 2 summarizes the observations. In Section 3 and 4, X-ray light curves and X-ray spectra obtained from Suzaku data are discussed. In Section 5, the de-

*email: bharti@aries.res.in 
pendence of X-ray luminosity on binary separation is investigated followed by conclusions in Section 6.

\section{Observations}

WR 25 has been observed by Suzaku on 10 occasions from August 2005 to July 2013 taking $\eta$ Carinae at the centre of the field of view of the X-ray Imaging Spectrometer (XIS). Chandra made one observation of WR 25 in August 2006. Collectively, the observations made by these two satellites from 2005 to 2013 at 11 different epochs were used for the present study. The data was analysed using standard packages of X-ray analysis and the latest version of calibration files for both satellites.

\section{X-ray Light Curves}

The background subtracted X-ray light curves, as observed by the front illuminated (FI) XIS units viz. XIS- 0 and XIS-3 onboard Suzaku, were added. The X-ray light curves obtained were folded using an ephemeris HJD = $2451598.0+207.85 \mathrm{E}$ (Gamen et al. 2006), where HJD = 2451598.0 corresponds to the zero phase in the folded light curves at the time of periastron passage. The folded X-ray light curves in the broad (0.3-10.0 keV), soft (0.3-2.0 keV) and hard (2.0-10.0 keV) energy bands are shown in panels (a), (b), and (c) of Fig. 1, respectively. The light curves in the individual energy bands show phase locked variability. The count rate drops suddenly after the periastron passage in the broad- and soft-band light curves and then increase again up to the phase $\sim 0.88$. However, in the hard energy band, the count rate decreased systematically while moving away from periastron, being minimum at apastron. The hardness ratio (HR), defined as (Hard-Soft)/(Hard+Soft) and shown in panel (d) of Fig. 1, reveals information about the spectral variations. The larger value of HR near phase $\sim 0.06$ indicates a harder spectrum at that phase.

\section{X-ray Spectra}

The X-ray spectra of WR 25 as observed by FI XIS-0 and XIS-3 were added and strong emission lines from various species were identified. The spectra were fitted using the models of Astrophysical Plasma Emission Code (APEC; Smith et al. 2001) in the X-ray spectral fitting package XSPEC. The form of model used for spectral fitting was PHABS(ISM)*PHABS(LOCAL)*(VAPEC+VAPEC) and the $\chi^{2}$ minimization method was applied to optimize the fit of the model to the data. While performing spectral fitting, abundances of $\mathrm{He}, \mathrm{C}$ and $\mathrm{N}$ were fixed at 2.27, 0.15 and 5.9 respectively (obtained by Crowther et al. 1995 from optical spectra of WR 25). The temperature for both components, the interstellar medium hydrogen column density $\left(N_{H}^{I S M}\right)$ as well as the abundances of various species were found to be almost constant at all the orbital phases and their average values were similar to those obtained by Pandey et al. (2014). Therefore, further spectral fitting was done by fixing these parameters to the values of Pandey et al. (2014). The local hydrogen column density $\left(N_{H}^{\text {local }}\right)$ and normalization constant for both the components were kept free. The emission measures (EMs), $E M_{1}$ and $E M_{2}$ corresponding to the cool and hot plasma components, respectively, were calculated from the associated normalization parameters. The X-ray fluxes of WR 25 at different orbital phases were estimated using the CFLUX model in XSPEC in the soft $(0.3-2.0 \mathrm{keV})$ and hard $(2.0-10.0 \mathrm{keV})$ energy bands. The luminosities in the soft $\left(L_{S}\right)$ and hard $\left(L_{H}\right)$ energy bands were derived from the corresponding flux values at a source distance of $3.24 \mathrm{kpc}$ (van der Hucht 2001). The variations of $E M_{1}, E M_{2}, N_{H}^{\text {local }}, L_{H}$ and $L_{S}$ with orbital phase are plotted in Fig. 2. 
The maximum values of $L_{H}$ and $L_{S}$ are found near the phase of 0.88 , close to the periastron passage. $L_{S}$ drops suddenly to the phase $\sim 0.06$ where $N_{H}^{\text {local }}$ is maximum. Furthermore, $N_{H}^{\text {local }}$ decreases to the phase $\sim 0.5$ with a corresponding increase in $L_{S}$. After that, $N_{H}^{\text {local }}$ as well as $L_{S}$ become almost constant until $L_{S}$ again increases near the periastron passage at a phase $\sim 0.88$. The minimum value of $L_{S}$ is obtained just after its maximum value that is 1.9 times more than the minimum value. However, $L_{H}$ decreases gradually from phase $\sim 0.06$ to the phase $\sim 0.5$ and again increases to the phase $\sim 0.88$, near the periastron passage. The maximum value of $L_{H}$ obtained is 1.8 times more than its minimum value. The EMs, $E M_{1}$ and $E M_{2}$ corresponding to the cool and hot plasma components, are also found to be phase dependent, being minimum at phase $\sim 0.5$ and maximum at phase $\sim 0.88$.

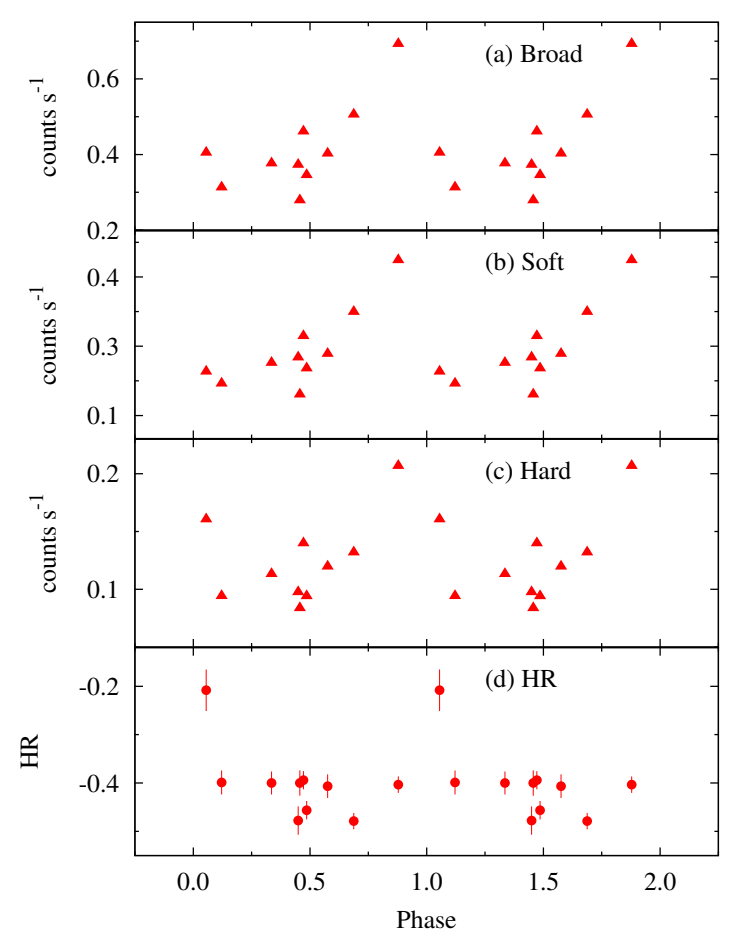

Figure 1: Top: Folded X-ray light curves in different energy bands: (a) broad (0.3-10.0 keV), (b) soft (0.3-2.0 keV), and hard (2.0-10.0 keV). Bottom: Folded values of the hardness ratio (d). In each panel, the reference epoch HJD = 2451598.0 corresponds to periastron passage.

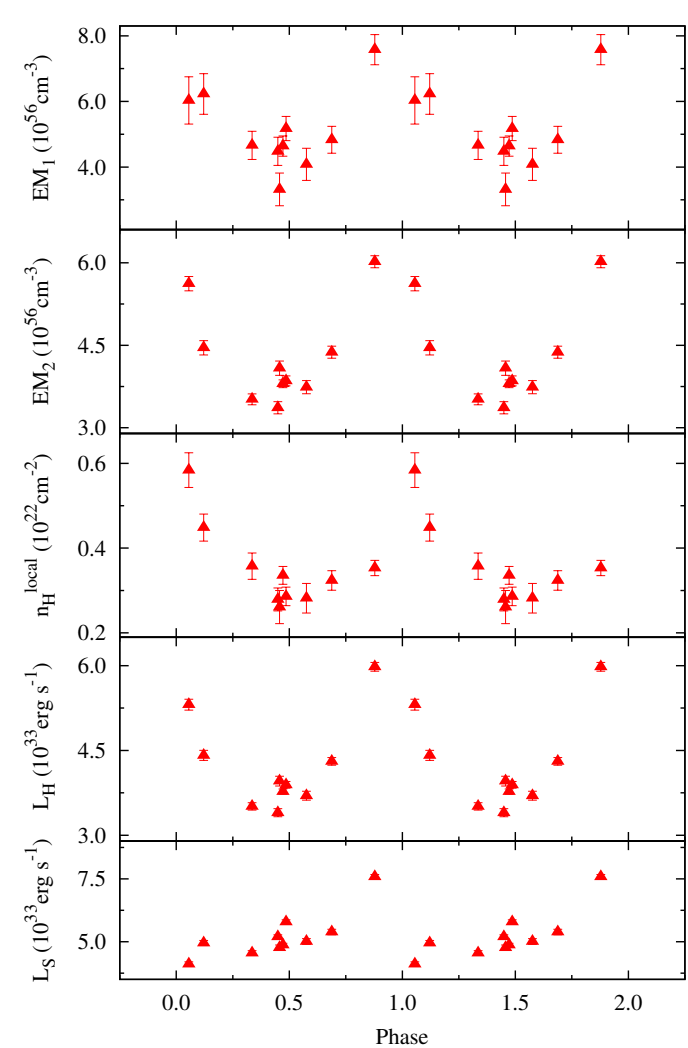

Figure 2: Spectral parameters as a function of orbital phase. In each panel, the reference epoch HJD = 2451598.0 corresponds to periastron passage.

\section{$5 \quad L_{X}$ versus binary separation}

The phase-locked variability in the X-ray light curves and spectral parameters of WR 25 could be a consequence of the changing binary separation in its eccentric orbit. The variation of X-ray luminosity in the soft $(0.3-2.0 \mathrm{keV})\left(L_{S}\right)$ and hard (2.0-10.0 keV) $\left(L_{H}\right)$ energy bands with the inverse of the normalized binary separation (the binary separation D normalized to the semi-major axis a), as observed by Chandra, Suzaku, Swift and XMM-Newton is shown in Fig. 3. The Swift and XMM-Newton luminosities were taken from Pandey et al. (2014). $L_{H}$ varies with the inverse of D: $L_{H}$ increases 
when D decreases (and vice versa) but there is a deviation from a linear trend. $L_{S}$ doesn't seem to follow a similar trend as $L_{H}$.

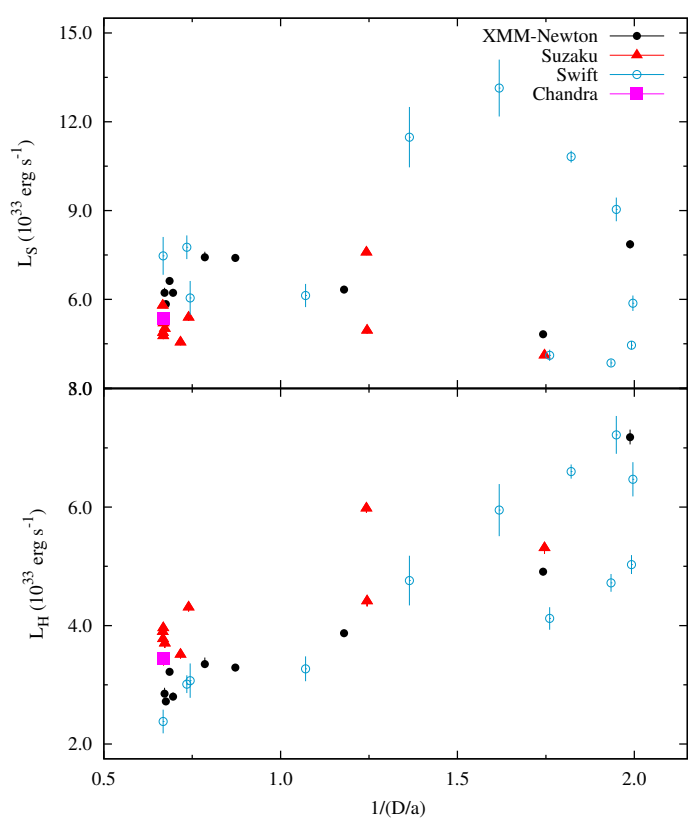

Figure 3: X-ray luminosity as a function of inverse of binary separation in soft and hard X-ray energy bands.

\section{Conclusions}

Using the data from Chandra and Suzaku, we have investigated the wind properties of WR 25. We found that the X-ray emission is stronger as the stars approach periastron in the $2.0-10.0 \mathrm{keV}$ energy band, while the emission is stronger in the $0.2-2.0 \mathrm{keV}$ energy band when they recede. A hysteresis of the intrinsic X-ray emission appears to be present when the luminosity is plotted against the binary separation. With the high-resolution spectrograph proposed for the 3.6-m Devasthal Optical Telescope (DOT), a continuous spectroscopic monitoring of colliding wind binaries could provide information about the wind properties at various orbital phases and help to get an in-depth understanding of their dynamics.

\section{Acknowledgements}

This research has made use of data obtained from the Suzaku satellite, a collaborative mission between the space agencies of Japan (JAXA) and the USA (NASA), as well as from Chandra Data Archive and the Chandra Source Catalog, and software provided by the Chandra X-ray Center (CXC) in the application packages CIAO, ChIPS, and Sherpa. 


\section{References}

Crowther P. A., Hillier D. J., Smith L. J. 1995, A\&A, 293, 403

Gamen R., Gosset E., Morrell N. et al. 2006, A\&A, 460, 777

Hamann W.-R., Gräfener G., Liermann A. 2006, A\&A, 457, 1015

Mitsuda K., Bautz M., Inoue H. et al. 2007, PASJ, 59, S1-S7

Pandey J. C., Pandey S. B., Karmakar S. 2014, ApJ, 788, 84

Pollock A. M. T., Corcoran M. F. 2006, A\&A, 445, 1093

Raassen A. J. J., van der Hucht K. A., Mewe R. et al. 2003, A\&A, 402, 653

Seward F. D., Chlebowski T. 1982, ApJ, 256, 530

Smith R. K., Brickhouse N. S., Liedahl D. A., Raymond J. C. 2001, ApJ, 556, 91

Stevens I. R., Blondin J. M., Pollock A. M. T. 1992, ApJ, 386, 265

van der Hucht K. A. 2001, NewAR, 45, 135

Weisskopf M. C., Tananbaum H. D., Van Speybroeck L. P., ODell S. L. 2000, Proc. SPIE Conf. Ser. Vol. 4012, X-Ray Optics, Instruments, and Missions III. SPIE, Bellingham, p. 2 